\title{
Trends in popularity of some morphological traits of purebred dogs in Australia
}

\author{
Kendy T. Teng ${ }^{1 *}$, Paul D. McGreevy², Jenny-Ann L. M. L. Toribio ${ }^{3}$ and Navneet K. Dhand ${ }^{3}$
}

\begin{abstract}
Background: The morphology of dogs can provide information about their predisposition to some disorders. For example, larger breeds are predisposed to hip dysplasia and many neoplastic diseases. Therefore, longitudinal trends in popularity of dog morphology can reveal potential disease pervasiveness in the future. There have been reports on the popularity of particular breeds and behavioural traits but trends in the morphological traits of preferred breeds have not been studied.

Methods: This study investigated trends in the height, dog size and head shape (cephalic index) of Australian purebred dogs. One hundred eighty-one breeds derived from Australian National Kennel Council (ANKC) registration statistics from 1986 to 2013 were analysed. Weighted regression analyses were conducted to examine trends in the traits by using them as outcome variables, with year as the explanatory variable and numbers of registered dogs as weights. Linear regression investigated dog height and cephalic index (skull width/skull length), and multinomial logistic regression studied dog size.
\end{abstract}

Results: The total number of ANKC registration had decreased gradually from 95,792 in 1986 to 66,902 in 2013. Both weighted minimal height $(p=0.014)$ and weighted maximal height $(p<0.001)$ decreased significantly over time, and the weighted cephalic index increased significantly $(p<0.001)$. The odds of registration of medium and small breeds increased by $5.3 \%$ and $4.2 \%$, respectively, relative to large breeds $(p<0.001)$ and by $12.1 \%$ and $11.0 \%$, respectively, relative to giant breeds $(p<0.001)$ for each 5 -year block of time.

Conclusions: Compared to taller and larger breeds, shorter and smaller breeds have become relatively popular over time. Mean cephalic index has increased, which indicates that Australians have gradually favoured breeds with shorter and wider heads (brachycephalic). These significant trends indicate that the dog morphological traits reported here may potentially influence how people select companion dogs in Australia and provide valuable predictive information on the pervasiveness of diseases in dogs.

Keywords: Purebred dogs, Dog popularity, Dog height, Dog size, Cephalic index, Brachycephalic, Disease predisposition, Australia

\section{Plain English Summary}

Some diseases in dogs are related to certain physical characteristics. For example, larger breeds have a higher risk of getting hip dysplasia and certain neoplastic diseases while breeds with wider and shorter heads, such as Pug and French bulldog, are more likely to experience

\footnotetext{
* Correspondence: kendy.teng@sydney.edu.au

${ }^{1}$ Faculty of Veterinary Science, School of Life and Environmental Sciences, The University of Sydney, JD Stewart Building (B01), Camperdown, NSW 2050, Australia

Full list of author information is available at the end of the article
}

breathing problems and dystocia. Therefore, if we know the trends in popularity of dogs of a certain morphology, we may be able to predict disease pervasiveness.

The study aimed to investigate the trends in the height, dog size and head shape of Australian purebred dogs. The numbers of dogs registered within the 181 breeds in Australian National Kennel Council (ANKC) every year from 1986 to 2013 were obtained and analysed.

The total number of ANKC registration had decreased from 95,792 in 1986 to 66,902 in 2013. Compared to 
taller and larger breeds, shorter and smaller breeds have become relatively popular over time. Also, the data suggest that Australians increasingly favour dogs with shorter and wider heads for whose welfare veterinarians often express concern $[1,2]$.

The results indicate that dog height, dog size and dog head shape may potentially influence how people select companion dogs in Australia and provide valuable predictive information on trends in disease prevalence, enabling the veterinary profession and industry to prepare for potential future caseloads.

\section{Background}

Breed predispositions to disease are well recognized [3]. Regardless of breed, the morphologies of dogs often imply an individual's predisposition to some disorders [4]. For example, hip dysplasia $[5,6]$, gastric dilatation volvulus $[7,8]$ and many neoplastic diseases [9] frequently occur in larger sized breeds of dogs. In contrast, smaller breeds of are predisposed to myxomatous mitral valve disease [10] and tracheal collapse [11-13]. Additionally, brachycephalic breeds are susceptible to several health concerns such as brachycephalic airway obstruction syndrome (BAOS) [14, 15], dystocia in dams [16], digestive disorders and multiple eye conditions [4].

People prefer different types of dogs and use various criteria for selecting their household dogs, which can be influenced by human lifestyle, cultural backgrounds, media exposure, education, etc. Therefore, changes in any of these may impact the decision making process in dog selection. For example, the purpose of keeping dogs has been shifting from specific functionalities to primarily companion in the western world and this would potentially have had impacts on people's choice of dogs [17]. Several studies have been conducted to understand the trends in the popularity of dogs and their reasons. A previous study on trends in the popularity of purebred dogs suggested that breeds became highly favoured mainly by chance without a specific trend [18]. It was also proposed that social influence (fashion) is the primary influence on the popularity of companion dog breeds, which is related to media exposure $[19,20]$, and that people showed no preference to breeds with sound health and good behaviours $[19,21]$. However, although the appearance and the size of the dog are often considered while selecting a companion dog, we are not aware of any research conducted to reveal the potential trends in the popularity of morphological traits of dogs.

This study was conducted to investigate potential trends in some morphological traits of Australian purebred dogs, including dog height, dog size and cephalic index (skull width divided by skull length), by analysing a 28-year-long (1986-2013) Australian National Kennel Council (ANKC) dog registration dataset (Additional file 1) [22].

\section{Methods}

\section{Data collection and management}

ANKC has recorded and published the registration numbers of each ANKC-recognised breed on their website each year since 1986 [22]. For every year from 1986 to 2013, the numbers of dogs registered for 181 breeds were drawn from the ANKC registration report. For some breeds, there were more than one observation due to the breeds' variation, such as Chihuahua, whose hair can be long or smooth; therefore, 204 observations (each breed and its variations) were independently included in the study.

Height and weight references of breeds were obtained from the ANKC studbook [23] or from the Encyclopedia of Dog Breeds (EoDB) [24], if not available in the studbook. Height is the distance from a dog's withers to the ground. The lowest and highest height values in one breed were chosen as the minimal and maximal heights for the breed, respectively, and the minimal and maximal weight were determined likewise. For example, as the minimal and maximal heights in male Saint Bernard are 70 and $90 \mathrm{~cm}$ and 65 and $80 \mathrm{~cm}$ for female, respectively, 65 and $90 \mathrm{~cm}$ were then considered as the minimal and maximal height values for Saint Bernard. The weight range for each breed was used to assign breeds to one of the four size groups, namely, small (less than $10 \mathrm{~kg}$ ), medium (10 kg to less than $25 \mathrm{~kg}$ ), large ( 25 to less than $40 \mathrm{~kg}$ ) and giant (40 kg and over) based on the widely applied criteria [25-27]. A breed belonged to a size group when its whole weight range located in the group; otherwise, it would be classified into the group whose range covered the average of minimal and maximal weight of the breed. Breeds with only one weight value were categorised according to the specified value. Cephalic index and standard deviations of both sexes for 80 breeds were drawn from the literature and calculated for each breed [28]. Larger cephalic index indicates the head shape of the breed is more brachycephalic, whereas breeds with smaller values have a more dolichocephalic shape of head. Characteristics and their source of the most popular 20 breeds in Australia in 2013 are presented in Table 1 and Additional file 2 lists the information of all ANKC-recognised breeds.

\section{Statistical methods}

Data were extracted into and cleaned in Microsoft Excel 2010 spreadsheets. Descriptive statistics were generated for the overall trends in the number of registrations and in each morphological trait in Microsoft Excel 2010. The means of minimal height, maximal height, minimal weight, maximal weight and cephalic index, weighted by the registration numbers, over the 28 years were calculated and plotted, as well as the proportions of registration number of each size group. Correlation between minimal height, maximal height, minimal weight and maximal weight were 
Table 1 The morphologies of the most popular 20 Australian National Kennel Council (ANKC)recognised breeds in 2013

\begin{tabular}{|c|c|c|c|c|c|c|}
\hline Breed & Source of Height & Height Range $(\mathrm{cm})$ & Source of Weight & Weight Range (kg) & Dog size & Cephalic Index $\left( \pm S D^{a}\right)$ \\
\hline American Staffordshire Terrier & ANKC & $(43,48)$ & EoDB & $(25.9,30.4)$ & Large & $67.40( \pm 3.34)$ \\
\hline Australian Cattle Dog & ANKC & $(43,51)$ & EoDB & $(15.9,20.4)$ & Medium & $61.60( \pm 8.75)$ \\
\hline Border Collie & ANKC & $(46,53)$ & EoDB & $(13.6,20.4)$ & Medium & $56.70( \pm 4.32)$ \\
\hline Boxer & ANKC & $(53,61)$ & EoDB & $(22.7,36.3)$ & Large & $66.75( \pm 6.01)$ \\
\hline British Bulldog & EoDB & $(30,38)$ & EoDB & $(18.1,22.7)$ & Medium & $86.60( \pm 4.34)$ \\
\hline Bull Terrier & EoDB & $(53,56)$ & EoDB & $(22.7,31.8)$ & Large & $55.60( \pm 10.90)$ \\
\hline Cavalier King Charles Spaniel & EoDB & $(30,33)$ & ANKC & $(5.4,8.2)$ & Small & $76.25( \pm 4.78)$ \\
\hline Cocker Spaniel & ANKC & $(38,41)$ & ANKC & $(13.0,14.5)$ & Medium & $48.85( \pm 4.12)$ \\
\hline French Bulldog & EoDB & $(28,33)$ & EoDB & $\left(N A^{b}, 12.7\right)$ & Medium & $101.55( \pm 2.42)$ \\
\hline German Shepherd Dog & ANKC & $(55,65)$ & ANKC & $(22.0,40.0)$ & Large & $50.40( \pm 8.62)$ \\
\hline Golden Retriever & ANKC & $(51,61)$ & EoDB & $(25.0,34.0)$ & Large & $56.05( \pm 3.54)$ \\
\hline Great Dane & EoDB & $(79,89)$ & ANKC & $(46.0,54.0)$ & Giant & $56.60( \pm 4.56)$ \\
\hline Jack Russell Terrier & ANKC & $(25,30)$ & ANKC & $(5.0,6.0)$ & Small & $61.45( \pm 2.75)$ \\
\hline Labrador Retriever & ANKC & $(55,57)$ & EoDB & $(25.0,36.3)$ & Large & $55.95( \pm 4.80)$ \\
\hline Poodle (Toy) & EoDB & $\left(N A^{b}, 25\right)$ & EoDB & $(1.8,3.6)$ & Small & $N A^{b}$ \\
\hline Pug & EoDB & $(25,28)$ & ANKC & $(6.3,8.1)$ & Small & $98.55( \pm 6.74)$ \\
\hline Rhodesian Ridgeback & ANKC & $(61,69)$ & EoDB & $(31.8,38.6)$ & Large & $50.45( \pm 3.10)$ \\
\hline Rottweiler & ANKC & $(56,68)$ & EoDB & $(36.3,61.2)$ & Giant & $63.55( \pm 2.95)$ \\
\hline Schnauzer (Miniature) & EoDB & $(30,36)$ & EoDB & $(5.9,6.8)$ & Small & $53.40( \pm 2.44)$ \\
\hline Staffordshire Bull Terrier & ANKC & $(36,41)$ & ANKC & $(11.0,17.0)$ & Medium & $76.15( \pm 6.32)$ \\
\hline
\end{tabular}

Legend: The morphologies of the 20 most popular dog breeds in Australian National Kennel Council (ANKC) registry in 2013, based on data sourced from the ANKC breed standards and the Encyclopedia of Dog Breeds (EoDB). Cephalic Index data were required from a peer-reviewed paper [28]. Characteristics of all ANKC-recognised breeds are presented in Additional file 2

${ }^{\mathrm{a} S D}$ : standard deviation; ${ }^{\mathrm{b}} \mathrm{NA}$ : missing data

calculated. To insure that the contribution of each observation is proportional to the number of registration, weighted regression analyses were then conducted to examine trends in each trait, using the trait as the outcome variable, year as the explanatory variable and numbers of registered dogs as weights to account for different numbers of registration of each breed each year. Linear regression investigated dog height and cephalic index and multinomial logistic regression studied dog size by using the SAS statistical program, 9.3th edition (SAS Institute, Cary, NC. USA). Normality and homoscedasticity were assessed by visual inspection of residual and residual-versus-fitted plots. A two sided $\mathrm{P}$-value $<0.05$ was considered statistically significant.

\section{Results}

\section{Descriptive statistics}

The total number of ANKC registration had decreased gradually from 95,792 in 1986 to 66,902 in 2013. A precipitous fluctuation in numbers registered between 1997-1998 was noted (Fig. 1a). The numbers of breeds and their variations which have at least one registry increased from 144 to 183 from 1986 to 2013 . While the majority of the height records were sourced from the
ANKC studbook $(n=140)$ and the remaining from the EoDB $(n=64)$, similar numbers of weight records were extracted from the ANKC studbook $(n=86)$ and the EoDB $(n=94)$. Standard Poodle had no maximal height value, whereas Smooth and Wire Fox Terrier, Toy Poodle, Shih Tzu, Tibetan Spaniel and Welsh Terrier had no minimal height values. In addition, 23 breeds and both variations of German Spitz (German Spitz Klein and German Spitz Mittel) were not classified into any size groups due to no weight records being available from the sources consulted. In total, there were 54 small breeds, 62 medium breeds, 42 large breeds, and 22 giant breeds for which data were used in this study.

The weighted mean of maximal height decreased from $50.0 \mathrm{~cm}$ in 1986 to $48.3 \mathrm{~cm}$ in 2013, and weighted mean of minimal height decreased from $43.6 \mathrm{~cm}$ in 1986 to $42.7 \mathrm{~cm}$ in 2013 (Figs. 1b and 2). Weighted mean of cephalic index increased from 57.7 in 1986 to 62.9 in 2013 (Fig. 1c). The proportions of small, medium and large breeds ranged between 0.26 to 0.36 in the 28 -year period, while the proportion of giant breed only ranged from 0.07 to 0.14 (Fig. 1d). The proportion of medium sized breeds steadily increased over time. 


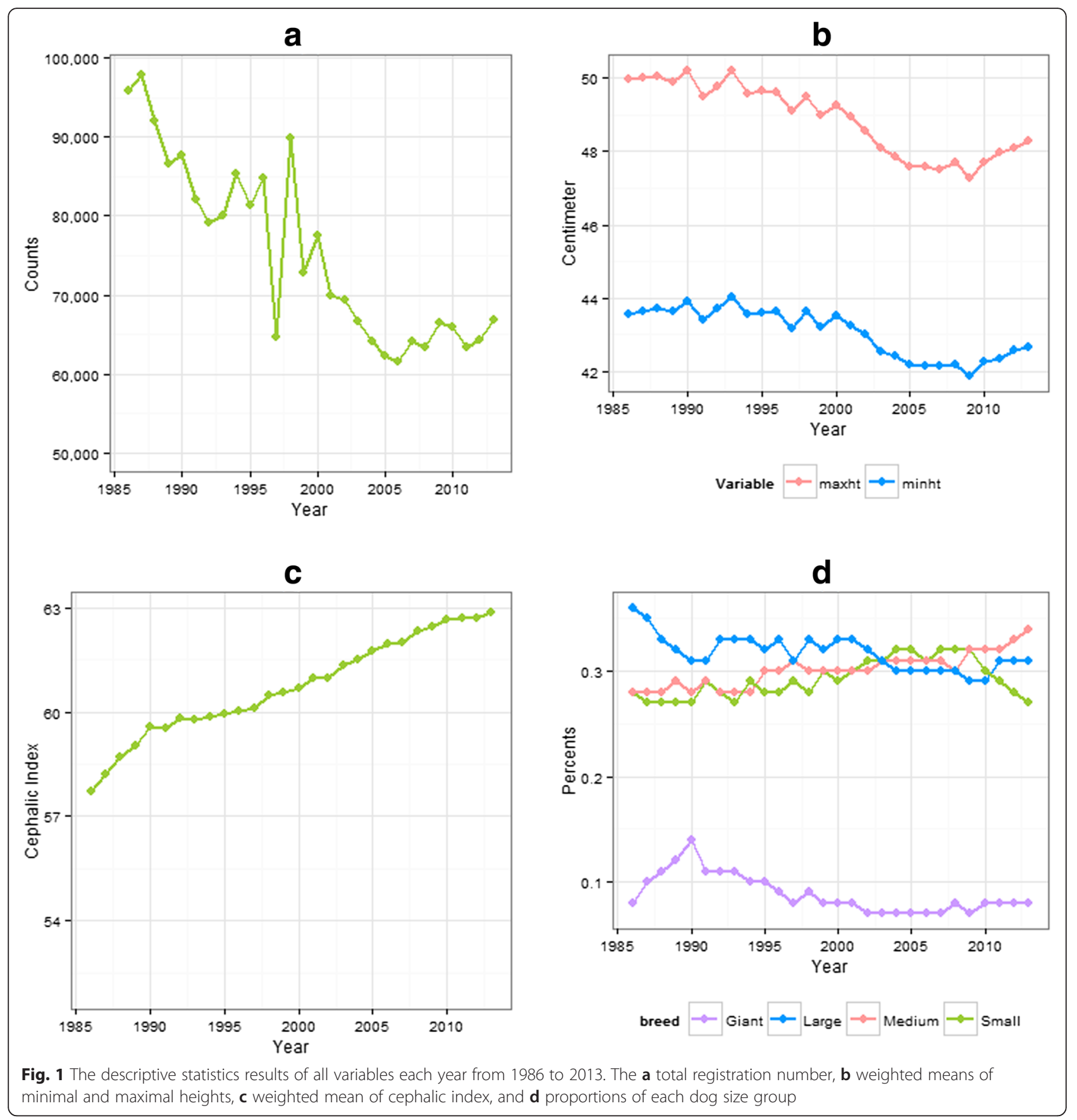

\section{Modelling results}

Correlations between minimal and maximal heights, and minimal and maximal weights were $0.98(p<0.001)$ and $0.96(p<0.001)$, respectively. Correlations between minimal height and weight, and maximal height and weight were $0.82(p<0.001)$ and $0.86(p<0.001)$, respectively.

The results suggested that both weighted minimal height and maximal height decreased significantly over time while the weighted cephalic index increased significantly during the period (Table 2). The multinomial logistic model results indicated that odds of registration of medium and small breeds increased by $5.3 \%$ and $4.2 \%$, respectively, relative to large breeds $(p<0.001)$ and by $12.1 \%$ and $11.0 \%$, respectively, compared to giant breeds $(p<0.001)$ (Table 3) for each 5-year block of time.

\section{Discussion}

This study reveals previously undocumented trends in morphological traits of purebred dogs in Australia by using the ANKC registry dataset. Shorter and smaller breeds, as 


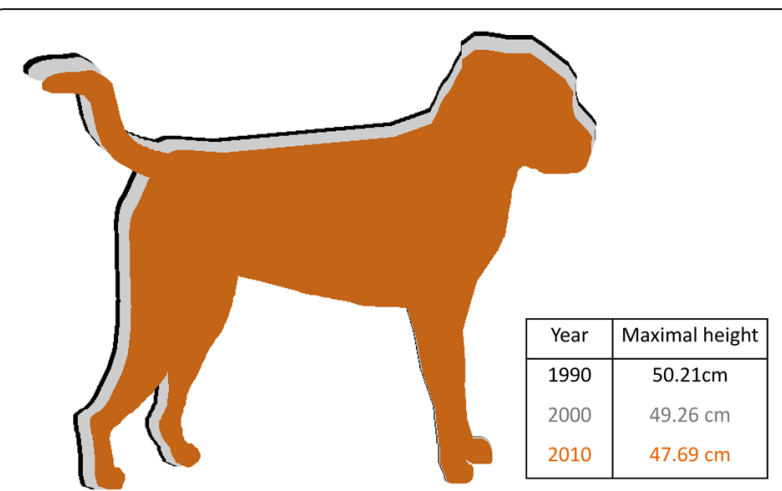

Fig. 2 The change of the mean dog maximal height in Australia every 10-year block of time

well as breeds with larger cephalic index, show growing popularity in ANKC registrations in the 28 years. These significant trends provide valuable predictive information on the pervasiveness of diseases in Australian companion dogs.

The total registration numbers have been continuously declining with a difference of about 30,000 over the 28 years. This can be due to decreases purely in numbers registered to ANKC, purebred dog numbers in Australia, dog numbers in Australia, or any combination of these factors. It is noted that the numbers of ANKC memberships have decreased from 54,590 in 1995 to 33,119 in 2013 [29]. However, new dog registrations would mostly depend on the number of newborn pedigree dogs rather than the number of ANKC members, and a breeder can have more than one breeding bitch. The profile of pedigree dog breeders may have been compromised by media focus on inherited disorders and puppy farms; this in combination with the promotion of adoptions may have reduced the demand for purebred dogs in Australia. In addition, the recent surges of popularity of designer breeds, those are crossbred with two different breeds of dogs, is noticed by veterinarians. The trend can also partially result from reduced dog population size in Australia. Pet industry figures indicate a decreasing trend from 1998 to 2009 [30], although there are also recent predictions of an upturn in Australian dog numbers [31].

Changes to human lifestyle, to dwelling types and to the roles of dogs in human societies can affect dog owners' decisions about dog acquisition including preferred breed or type. From 1995 to 2010, the proportion of single separate house purchases (excluding semidetached/row and townhouse/terrace house) decreased and flat/unit/apartment purchases increased among first home buyers with a mortgage in Australia [32], which may indicate that space available for dogs would also have shrunk. Moreover, the major purposes of dog ownership nowadays have changed from certain functionalities such as hunting and guarding properties, for which dogs are more likely to be larger, to purely companionship, a purpose which can be fulfilled by dogs of various size [17]. These may be two of the many possible explanations of trends in dog height and dog size observed in the current study.

The results show that breeds with a larger cephalic index have steadily become more popular, which indicates that Australians have gradually favoured dogs with shorter and wider heads (brachycephalic) more than those with longer and thinner heads (dolichocephalic). The brachycephaly boom seems to be worldwide. In agreement with our results, brachycephalic breeds such as English Bulldogs, French Bulldogs, Boxers and Pugs, have been becoming increasingly popular in the United Kingdom (UK) over recent years [33], and the numbers of Bulldogs and French bulldogs registered with the American Kennel Club have increased by $69 \%$ and $476 \%$, respectively, in the past decade [34]. The typical skull shape of a breed often aligns with the breed's original purpose. For example, medium and large size brachycephalic breeds have stronger bite force [35], which seems to align with their common historic role in baiting and fighting context [36]. On the other hand, a dolichocephalic morphology is associated with a breed's ability as a visual hunter [37]. However, as functionality has become a minor incentive

Table 2 Weighted linear regression model results for the trends in dog height and cephalic index of Australian National Kennel Council (ANKC) recognised breed

\begin{tabular}{lllllrr}
\hline Outcome variable & Parameter & $\mathrm{b}$ & S.E. & $95 \% \mathrm{Cl}^{\mathrm{a}}$ & $\mathrm{t}$-value & $P$-value \\
\hline Maximal Height & Intercept & 50.62 & 0.48 & $(49.68,51.56)$ & 105.60 & $<0.001$ \\
& Year & -0.10 & 0.03 & $(-0.16,-0.04)$ & -3.34 & $<0.001$ \\
Minimal Height & Intercept & 44.04 & 0.43 & $(43.20,44.89)$ & 102.33 & -2.46 \\
& Year & -0.07 & 0.03 & $(-0.12,-0.01)$ & 0.001 \\
Cephalic Index & Intercept & 58.18 & 0.47 & $(57.26,59.11)$ & 123.63 & 0.014 \\
& Year & 0.17 & 0.03 & $(0.16,0.23)$ & 5.83 & $<0.001$ \\
\hline
\end{tabular}

Legend: Weighted linear regression model results with the maximal height, minimal height and cephalic index of the breeds ( $n=204$ ) in Australian National Kennel Council (ANKC) as outcome variables, year $(1986-2013)$ as the predictor and numbers of registered dogs each breed each year in ANKC as weights over the 28 years

${ }^{\mathrm{a}} 95 \% \mathrm{Cl}: 95 \%$ confidence interval 
Table 3 Weighted multinominal regression model results for the trend in dog size of Australian National Kennel Council (ANKC) recognised breeds

\begin{tabular}{llllllll}
\hline Outcome variable & Category & Intercept & $b$ & S.E. & $P$-value & OR & $95 \% \mathrm{Cl}^{\mathrm{b}}$ \\
\hline Dog size & Giant & - & 0 & - & - & 1 & - \\
& Small & 0.894 & 0.104 & 0.002 & $<.001$ & 1.110 & $(1.1061 .113)$ \\
& Medium & 0.894 & 0.114 & 0.002 & $<.001$ & 1.121 & $(1.117,1.125)$ \\
& Large & 1.010 & 0.063 & 0.002 & $<.001$ & 1.065 & $(1.062,1.068)$ \\
\hline
\end{tabular}

Legend: Weighted multinominal regression model results with the dog size as outcome variable, year (1986 - 2013) as the predictor and numbers of registered dogs each breed each year in Australian National Kennel Council registry as weights over the 28 years

${ }^{a}$ OR: odds ratio; ${ }^{b} 95 \% \mathrm{Cl}: 95 \%$ confidence interval

to acquire dogs, the popularity of breeds with larger cephalic index may have two possible causes instead of functionality. Firstly, the neotenic appearance of brachycephalic dogs may account for the popularity [38]. Many research studies have shown that the infantile facial features stimulate affective and caretaking behavioural responses in human adults, which has the evolutionary benefits of increasing the survival of the vulnerable individuals [39-42]. These cute features, defined by Konrad Lorenz (1943), are called "baby schema" [43], including large head, round face, chubby cheeks, high and protruding forehead, big eyes, small nose and mouth, etc. Interestingly, baby schema effect has been observed not merely in human infant but also cross species [44-46]. The head of brachycephalic dogs is characterised by a round and short face, open orbitae, a small and short nose, which accord with the baby schema features [38]. Therefore, baby schema effect may explain the increasing popularity of brachycephalic breeds. Secondly, a flux in perceived aesthetics may be responsible for the phenomenon. It has been confirmed that human behaviours and preferences can be contagious without rationale [47]. One study endorses this theory by demonstrating that fads play a major factor in choosing the breed of companion dogs [19].

Accompanying the trends in the prevalence of the morphological traits revealed in this study, we predict corresponding changes in the patterns of disease occurrence in dogs in Australia: diseases among smaller breeds and brachycephalic breeds are expected to be seen increasingly by the veterinary profession in Australia. The predicted increase in veterinary observation of diseases that predominantly affect these types of dogs can be tracked by examining and analysing electronic patient health records from primary care veterinary clinics longitudinally.

With the increase of smaller and brachycephalic dogs, conditions leading to mortality in small breeds (urogenital diseases, degenerative diseases, metabolic diseases) will potentially be seen more, compared to those that have an increased risk of death in larger breeds, such as diseases of musculoskeletal and gastrointestinal systems and many neoplastic diseases [48]. Through reviewing the literature, we have identified 13 common diseases in smaller breeds, compared to 25 diseases in larger breeds listed in the Additional file 3. In larger breeds, $28 \%$ of the diseases are musculoskeletal, $20 \%$ are nervous/sensory, $16 \%$ are cardiovascular, and $36 \%$ belong to the rest organ systems. In contrast, in smaller breeds, no clusters of diseases of specific organ systems have been noticed. Among the dog-size predisposed diseases, patellar luxation (PL), portosystemic shunt (PSS) and mammary tumour (MT) show different forms of predispositions in smaller and larger breeds. While PL was originally recognised as a condition to which smaller breeds were predisposed [49], the occurrence in larger breeds appears to be increasing $[50,51]$. Medial PLs are the predominant condition regardless of dog size [52], whereas the lateral form is reported more frequently in larger breeds [51, 53]. PSS is generally more prevalent in smaller breeds [54-56], especially in the form of extrahepatic PSSs [54, 57]. In contrast, intrahepatic PSS cases are seen more commonly in larger breeds $[54,58,59]$. Although MT is more frequently seen in smaller breeds of dogs [60], the MTs with greater malignancy [61] and thus a more profound effect on life expectancy are encountered among larger breeds [60]. We would like to acknowledge that the common diseases and disease occurrence may not be the same in different continents/countries due to the divergent gene pool although many of the disease predispositions are commonly recognised worldwide.

Concerns for the welfare of brachycephalic dogs have been highlighted recently and, as reported by our results and the literature, this issue is likely to become an increasing concern for veterinarians and dog owners in Australia and worldwide. In New Zealand, 4 of the top 5 dog breeds considered by veterinarians to be unsuitable for continued breeding due to compromised health and welfare are brachycephalic breeds [1]. The life expectancy is estimated 4 years lower in highly brachycephalic breeds than those not (8.6 years vs 12.7 years) [62] BAOS, resulting in mild to life-threatening respiratory dysfunction [14], has received attention in the UK following the growing popularity of brachycephalic breeds in that country [33, 63]. However, a UK study showed that approximately half of the owners of BAOS affected dogs seem unaware of BAOS in their dogs [64], which 
indicates that they did not make informed decisions when they purchased them, that dogs might not receive necessarily medical treatment when BAOS emerges, and that the use of affected dogs might persist in breeding programmes. Cephalopelvic disproportion between whelps and dams is thought to be responsible for dystocia in brachycephalic dogs and to lead to inevitable caesarean section [65]. One paper reports caesarean section being performed to deliver over $80 \%$ of litters for registered pedigree bitches of Boston Terrier, English Bulldog and French Bulldog in the UK [66], and the biggest Swedish insurance company applies special rules for reimbursement associated with caesarean section to these three breeds [67]. Additionally, there is some suggestion that pregnant brachycephalic dams often receive caesarean section before natural parturition begins [66]. Other brachycephalic-predisposed conditions include mast cell tumours [68], chemoreceptor system neoplasms [69-71], hydrocephalus [72] and multiple digestive, ocular and dermatological disorders $[4,73]$.

This is the first trend study in the popularity of canine morphological traits other than those determined solely by breed. By fitting model of random genetic drift, Herzog suggests that the randomness of fashion largely explains the popularity of dog breeds $[18,19]$. Another study shows no correlation between popularity and the longevity or the desirable behavioural tendencies of breeds [21]. As our results show linear relationships between certain morphological traits of dogs and time, this may suggest that the preference for morphological features of dogs may be embedded in social changes and trends, such as urbanisation and pursuit of cuteness, which influence people's criteria for selecting household dogs. By knowing that dog size is often a consideration while choosing a dog along with our results [74], it is reasonable to conclude that dog height and dog size may potentially be one of the major considerations in decision making process of selecting companion dogs in Australia. However, we would also like to acknowledge that there may be other unmeasured factors influencing trends in dog numbers over time that we cannot capture from the data we have.

This study has a number of possible limitations that we wish to acknowledge. Firstly, although the ANKC dog registration dataset has high data integrity with few missing data, the results are highly representative of ANKC-registered dogs, and so may not truly represent either the purebred population or general dog population in Australia. The ANKC estimates that $16.5 \%$ of newborn puppies in Australia in 2014 were from ANKC [75]. That said, since the ANKC is the leading kennel club in Australia, it is plausible that the composition of dog breeds in the Australian purebred population reflects those registered with ANKC. However, this would be based on the assumption that purebred dogs outside of ANCK also strictly follow the ANKC breed standards, which may not be true. Secondly, not all the height and weight records are representative of Australian purebred dogs since some are derived from EoDB, where most standards are from the American Kennel Club. Additionally, both of these sources adopt various standardising methods for different breeds, which limits the consistency of our data. For instance, while most breeds have minimal and maximal height restriction for both sexes, some have a height range for the breed or only a mean height. Thirdly, even though dog size groups are commonly used for research and in everyday veterinary practice, no universal dog sizing criteria can be found from the literature. For the current study, we classified dog size according to dog weight, as is standard practice in academic research and is considered a better predictor of lifespan than dog height [76]. Lastly, although significant linear trends appear in the changes of the morphological traits over time, we could only postulate about the best explanations for the identified trends but were not be able to test the causality in the current study.

\section{Conclusions}

This study identifies that, over the 28-year period (1986-2013), shorter and smaller breeds became relatively popular, compared to taller and larger breeds, and the mean cephalic index increased, suggesting that Australians are tending to prefer breeds with a wider and shorter head. These significant trends indicate that the dog morphological traits reported here may potentially influence how people select companion dogs in Australia and suggest valuable predictive information on the pervasiveness of diseases, enabling the veterinary profession and industry to prepare for potential future caseloads. It would be interesting to examine similar datasets from other countries.

\section{Additional files}

Additional file 1: The registration numbers of all Australian National Kennel Council (ANKC) recognised breeds in ANKC registry from 1986 to 2013. (XLSX $42 \mathrm{~kb}$ )

Additional file 2: The morphologies of all Australian National Kennel Council (ANKC) recognised breeds, based on data sourced from the ANKC breed standards and the Encyclopedia of Dog Breeds (EoDB). Cephalic Index data were derived from a peer-reviewed paper [28]. Characteristics of the most popular 20 breeds in 2013 are presented in Table 1. (DOCX $44 \mathrm{~kb}$ )

Additional file 3: Table S1. Disease predispositions in larger breeds of dogs. Table S2 Disease predispositions in smaller breeds of dogs. (DOCX 91 kb)

\section{Abbreviations}

BAOS: Brachycephalic Airway Obstruction Syndrome; ANKC: Australian National Kennel Council; EoDB: Encyclopedia of Dog Breeds; UK: United Kingdom; PL: Patellar Luxation; PSS: Portosystemic Shunts; MT: Mammary Tumours. 


\section{Competing interests}

The authors declare that they have no competing interests.

\section{Authors' contributions}

KTT is the primary author for this study and was involved in the study design, data collection and management, data analysis, result interpretation and drafting of the manuscript. PDM contributed to the conception of the work and helped to revise the manuscript. JLMLT was involved in result interpretation, drafting and revising the manuscript. NKD contributed to the conception and design of the work, data analysis, and helped to revise the manuscript. All authors read and approved the final manuscript.

\section{Acknowledgements}

The authors acknowledge contribution from Mao-Hsuan Chang, Wan-Chu Hung and Chen-I Liu for assisting in preparation of the Additional file 2.

\section{Author details}

${ }^{1}$ Faculty of Veterinary Science, School of Life and Environmental Sciences, The University of Sydney, JD Stewart Building (B01), Camperdown, NSW 2050, Australia. ${ }^{2}$ Faculty of Veterinary Science, School of Life and Environmental Sciences, The University of Sydney, R.M.C. Gunn Building (B19), Camperdown, NSW 2050, Australia. ${ }^{3}$ Faculty of Veterinary Science, School of Life and Environmental Sciences, The University of Sydney, JL Shute Building (C01A), 425 Werombi Road, Camden, NSW 2570, Australia

\section{Received: 29 October 2015 Accepted: 3 March 2016}

\section{Published online: 05 April 2016}

\section{References}

1. Farrow T, Keown AJ, Farnworth MJ. An exploration of attitudes towards pedigree dogs and their disorders as expressed by a sample of companion animal veterinarians in New Zealand. N Z Vet J. 2014;62(5):267-73.

2. Buckland EL, Corr SA, Abeyesinghe SM, Wathes CM. Prioritisation of companion dog welfare issues using expert consensus. Anim Welf. 2014;23(1):39-46.

3. McGreevy PD, Nicholas FW. Some practical solutions to welfare problems in dog breeding. Anim Welf. 1999;8(4):329-41.

4. Asher L, Diesel G, Summers JF, McGreevy PD, Collins LM. Inherited defects in pedigree dogs. Part 1: Disorders related to breed standards. Vet J. 2009;182(3):402-11.

5. Lust G, Rendano VT, Summers BA. Canine hip dysplasia: concepts and diagnosis. J Am Vet Med Assoc. 1985;187(6):638-40.

6. Smith GK, Mayhew PD, Kapatkin AS, McKelvie PJ, Shofer FS, Gregor TP. Evaluation of risk factors for degenerative joint disease associated with hip dysplasia in German Shepherd Dogs, Golden Retrievers, Labrador Retrievers, and Rottweilers. J Am Vet Med Assoc. 2001;219(12):1719-24.

7. Glickman LT, Glickman NW, Perez CM, Schellenberg DB, Lantz GC. Analysis of risk factors for gastric dilatation and dilatation-volvulus in dogs. J Am Vet Med Assoc. 1994:204(9):1465-71.

8. Glickman LT, Glickman NW, Schellenberg DB, Raghavan M, Lee T. Non-dietary risk factors for gastric dilatation-volvulus in large and giant breed dogs. J Am Vet Med Assoc. 2000;217(10):1492-9.

9. Kelsey JL, Moore AS, Glickman LT. Epidemiologic studies of risk factors for cancer in pet dogs. Epidemiol Rev. 1998;20(2):204-17.

10. Parker HG, Kilroy-Glynn P. Myxomatous mitral valve disease in dogs: does size matter? J Vet Cardiol. 2012:14(1):19-29.

11. Done S, Claton-Jones D, Price E. Tracheal collapse in the dog: a review of the literature and report of two new cases. J Small Anim Pract. 1970;11(11):743-50.

12. Johnson L. Tracheal collapse: diagnosis and medical and surgical treatment. Vet Clin North Am Small Anim Pract. 2000;30(6):1253-66.

13. White R, Williams J. Tracheal collapse in the dog-is there really a role for surgery? A survey of 100 cases. J Small Anim Pract. 1994;35(4):191-6.

14. Lorinson D, Bright RM, White RAS. Brachycephalic airway obstruction syndrome - A review of 118 cases. Canine Pract. 1997;22(5-6):18-21.

15. Torrez CV, Hunt GB. Results of surgical correction of abnormalities associated with brachycephalic airway obstruction syndrome in dogs in Australia. J Small Anim Pract. 2006:47(3):150-4.

16. Pretzer SD. Medical management of canine and feline dystocia. Theriogenology. 2008;70(3):332-6.

17. Stafford K. The welfare of dogs, vol. 4. Springer Science \& Business Media. 2006

18. Herzog HA, Bentley RA, Hahn MW. Random drift and large shifts in popularity of dog breeds. Proc R Soc B-Biol Sci. 2004;271:S353-6.
19. Herzog H. Forty-two thousand and one dalmatians: Fads, social contagion, and dog breed popularity. Soc Anim. 2006:14(4):383-97.

20. Ghirlanda S, Acerbi A, Herzog H: Dog movie stars and dog breed popularity: a case study in media influence on choice. PLoS One 2014; 9(9):e106565. doi: 10.1371/journal.pone.0106565.

21. Ghirlanda S, Acerbi A, Herzog H, Serpell JA. Fashion vs. function in cultural evolution: the case of dog breed popularity. PLoS One. 2013;8(9):6.

22. Australian National Kennel Council. National animal registration analysis 1986-2013. 2013.

23. Australian National Kennel Council: Breed standard. http://ankc.org.au/ Breed/Index. Access 28 Jan 2015.

24. Coile DC. Encyclopedia of dog breeds. Hauppauge: Barron's Educational Series; 2005.

25. Eukanuba: The puppy guide - get him ready for an extraordinary start. 2014; 42-43.

26. Gandini G, Cizinauskas S, Lang J, Fatzer R, Jaggy A. Fibrocartilaginous embolism in 75 dogs: clinical findings and factors influencing the recovery rate. J Small Anim Pract. 2003;44(2):76-80

27. Royal Canin. Size-based nutrition. 2013. http://www.royalcanin.com.au/ puppy-dog/dogs/size-based-nutrition. Accessed 9 Jun 2015

28. Carrasco JJ, Georgevsky D, Valenzuela M, McGreevy PD. A pilot study of sexual dimorphism in the head morphology of domestic dogs. J Vet Behav. 2014;9(1):43-6.

29. Australian National Kennel Council. ANKC LTD membership statistics. 2014.

30. Australian Companion Animal Council. Contribution of the pet care industry to the Australian economy. 7th ed. 2010. p. 13.

31. Baguley J. An analysis of the demand for and revenue from companion animal veterinary services in Australia between 1996 and 2026 using industry revenue data and household census and pet ownership data and forecasts. Aust Vet J. 2011:89(9):352-9.

32. Australian Bureau of Statistics: 4130.0 - Housing occupancy and costs, 2007-08. 2009. http://www.abs.gov.au/AUSSTATS/abs@.nsf/Previousproducts/ 4130.0Feature\%20Article12007-08?opendocument\&tabname=Summary\& prodno $=4130$.0\&issue $=2007-08 \&$ num $=\&$ view. Accessed 12 Apr 2015.

33. Emmerson T. Brachycephalic obstructive airway syndrome: a growing problem. J Small Anim Pract. 2014:55(11):543-4.

34. Fasanella FJ, Shivley JM, Wardlaw JL, Givaruangsawat S. Brachycephalic airway obstructive syndrome in dogs: 90 cases (1991-2008). J Am Vet Med Assoc. 2010;237(9):1048-51.

35. Ellis JL, Thomason J, Kebreab E, Zubair K, France J. Cranial dimensions and forces of biting in the domestic dog. J Anat. 2009;214(3):362-73.

36. Collins T, Martin J, Vamplew W. Encyclopedia of traditional British rural sports: Psychology Press. 2005.

37. Schoenebeck JJ, Ostrander EA. The Genetics of Canine Skull Shape Variation Genetics. 2013:193(2):317-25.

38. Nöller C, Hueber J, Aupperle H, Seeger J, Oechtering T: New aspects of brachycephalia in dogs \& cats basics: insights into embryology, anatomy and pathophysiology. In: ACVIM Forum: 2008; San Antonio, Texas, USA; 2008: 713-715

39. Brosch T, Sander D, Scherer KR. That baby caught my eye... attention capture by infant faces. Emotion. 2007;7(3):685-9.

40. Luo LZ, Li H, Lee K. Are children's faces really more appealing than those of adults? Testing the baby schema hypothesis beyond infancy. J Exp Child Psychol. 2011:110(1):115-24.

41. Luo L, Ma X, Zheng X, Zhao W, Xu L, Becker B, Kendrick KM. Neural systems and hormones mediating attraction to infant and child faces. Front Psychol. 2015;6:970.

42. Glocker ML, Langleben DD, Ruparel K, Loughead JW, Gur RC, Sachser N. Baby Schema in Infant Faces Induces Cuteness Perception and Motivation for Caretaking in Adults. Ethology. 2009;115(3):257-63.

43. Lorenz K. Die angeborenen Formen möglicher Erfahrung. Z Tierpsychol. 1942; 5(2):235-409.

44. Lehmann V, Veld EMJ H i't, Vingerhoets AJJM. The human and animal baby schema effect: Correlates of individual differences. Behav Process. 2013:94:99-108.

45. Borgi M, Cogliati-Dezza I, Brelsford V, Meints K, Cirulli F. Baby schema in human and animal faces induces cuteness perception and gaze allocation in children. Front Psychol. 2014;5:411.

46. Kruger DJ. Non-Mammalian Infants Requiring Parental Care Elicit Greater Human Caregiving Reactions Than Superprecocial Infants Do. Ethology. 2015;121(8):769-74.

47. Marsden P. Memetics and social contagion: Two sides of the same coin JoM-EMIT. 1998;2(2):171-85. 
48. Fleming JM, Creevy KE, Promislow DEL. Mortality in North American dogs from 1984 to 2004: an investigation into age-, size-, and breed-related causes of death. J Vet Intern Med. 2011;25(2):187-98.

49. Priester WA. Sex, size, and breed as risk factors in canine patellar dislocation. J Am Vet Med Assoc. 1972;160(5):740-2.

50. Piermattei DL, Flo GL, DeCamp CE: Brinker, Piermattei, and Flo's handbook of small animal orthopedics and fracture repair. Elsevier Saunders; 2006

51. Hayes A, Boudrieau R, Hungerford L. Frequency and distribution of medial and lateral patellar luxation in dogs: 124 cases (1982-1992). J Am Vet Med Assoc. 1994;205(5):716-20.

52. Gibbons SE, Macias C, Tonzing MA, Pinchbeck GL, McKee WM. Patellar luxation in 70 large breed dogs. J Small Anim Pract. 2006;47(1):3-9.

53. Kalff S, Butterworth S, Miller A, Keeley B, Baines S, McKee W. Lateral patellar luxation in dogs: a retrospective study of 65 dogs. Vet Comp Orthop Traumatol. 2014;27(2):130-4.

54. Hunt GB. Effect of breed on anatomy of portosystemic shunts resulting from congenital diseases in dogs and cats: a review of 242 cases. Aust Vet J. 2004;82(12):746-9.

55. Tobias KM, Rohrbach BW. Association of breed with the diagnosis of congenital portosystemic shunts in dogs: 2,400 cases (1980-2002). J Am Vet Med Assoc. 2003;223(11):1636-9.

56. Winkler JT, Bohling MW, Tillson DM, Wright JC, Ballagas AJ. Portosystemic shunts: diagnosis, prognosis, and treatment of 64 cases (1993-2001). J Am Anim Hosp Assoc. 2003;39(2):169-85.

57. Worley DR, Holt DE. Clinical outcome of congenital extrahepatic portosystemic shunt attenuation in dogs aged five years and older: 17 cases (1992-2005). J Am Vet Med Assoc. 2008;232(5):722-7.

58. White R, Burton C, McEvoy F. Surgical treatment of intrahepatic portosystemic shunts in 45 dogs. Vet Rec. 1998;142:358-65.

59. Papazoglou LG, Monnet E, Seim HB. Survival and prognostic indicators for dogs with intrahepatic portosystemic shunts: 32 cases (1990-2000). Vet Surg. 2002;31(6):561-70.

60. Sorenmo KU, Worley DR, Goldschmidt MH. 27 - Tumors of the Mammary Gland. In: Withrow SJ, Vail DM, Page RL, editors. Withrow and MacEwen's Small Animal Clinical Oncology. 5th ed. Saint Louis: W.B. Saunders; 2013. p. 538-56.

61. Dobson JM: Breed-predispositions to cancer in pedigree dogs. Int Sch Res Notices 2013;(2013): 1-23. doi: 10.1155/2013/941275.

62. O'Neill D, Jackson C, Guy J, Church D, McGreevy P, Thomson P, Brodbelt D. Epidemiological associations between brachycephaly and upper respiratory tract disorders in dogs attending veterinary practices in England. Canine Genet Epidemiol. 2015;2(1):1-10.

63. Elliott $\mathrm{V}$. Leading vet calls for pugs and bulldogs ban because the pedigree dogs often struggle to breathe. 2012. http://www.dailymail.co.uk/news/ article-2106592/Leading-vet-calls-pugs-bulldogs-ban-pedigree-dogsstruggle-breathe.html. Accessed 13 May 2015.

64. Packer RMA, Hendricks A, Burn CC. Do dog owners perceive the clinical signs related to conformational inherited disorders as 'normal' for the breed? A potential constraint to improving canine welfare. Anim Welf. 2012;21:81-93.

65. Johnson CA. Disorders of Pregnancy. Vet Clin North Am Small Anim Pract 1986;16(3):477-82.

66. Evans KM, Adams VJ. Proportion of litters of purebred dogs born by caesarean section. J Small Anim Pract. 2010;51(2):113-8.

67. BergstrÖM A, NØDtvedt ANE, Lagerstedt A-S, Egenvall A. Incidence and Breed Predilection for Dystocia and Risk Factors for Cesarean Section in a Swedish Population of Insured Dogs. Vet Surg. 2006:35(8):786-91.

68. London CA, Thamm DH. 20 - Mast Cell Tumors. In: Withrow SJ, Vail DM, Page RL, editors. Withrow and MacEwen's Small Animal Clinical Oncology. 5th ed. Saint Louis: W.B. Saunders; 2013. p. 335-55.

69. Hayes HM. An hypothesis for the aetiology of canine chemoreceptor system neoplasms, based upon an epidemiological study of 73 cases among hospital patients. J Small Anim Pract. 1975;16(1-12):337-43.

70. Patnaik AK, Liu SK, Hurvitz Al, McClelland AJ. Canine chemodectoma (extra-adrenal paragangliomas)-a comparative study. J Small Anim Pract. 1975;16(1-12):785-801.

71. Owen TJ, Bruyette DS, Layton CE. Chemodectoma in dogs. Compend Contin Educ Pract Vet. 1996;45(40):253-6.

72. Dewey CW. A practical guide to canine and feline neurology. Hoboken: Wiley; 2013.

73. Pratschke K. Current thinking about brachycephalic syndrome: more than just airways. Companion Anim. 2014;19(2):70-8.
74. Garrison L, Weiss E. What Do People Want? Factors People Consider When Acquiring Dogs, the Complexity of the Choices They Make, and Implications for Nonhuman Animal Relocation Programs. J Appl Anim Welf Sci. 2015;18(1):57-73.

75. Australian National Kennel Council. A forensic view of puppy breeding in Australia. 2015.

76. Greer KA, Canterberry SC, Murphy KE. Statistical analysis regarding the effects of height and weight on life span of the domestic dog. Res Vet Sci. 2007:82(2):208-14

\section{Submit your next manuscript to BioMed Central and we will help you at every step:}

- We accept pre-submission inquiries

- Our selector tool helps you to find the most relevant journal

- We provide round the clock customer support

- Convenient online submission

- Thorough peer review

- Inclusion in PubMed and all major indexing services

- Maximum visibility for your research

Submit your manuscript at www.biomedcentral.com/submit

) Biomed Central 